\title{
A Note on Sparse Random Graphs and Cover Graphs* $^{*}$
}

\author{
Tom Bohman ${ }^{\dagger} \quad$ Alan Frieze B $^{\ddagger} \quad$ Miklós Ruszinkó $^{\S}$ \\ Lubos Thoma \\ Department of Mathematical Sciences \\ Carnegie Mellon University \\ Pittsburgh, PA 15213, USA \\ tbohman@andrew.cmu.edu, alan@random.math.cmu.edu \\ ruszinko@andrew.cmu.edu, thoma@qwes.math.cmu.edu \\ Submitted: December, 1999; Accepted: March 24, 2000.
}

\begin{abstract}
It is shown in this note that with high probability it is enough to destroy all triangles in order to get a cover graph from a random graph $G_{n, p}$ with $p \leq \kappa \log n / n$ for any constant $\kappa<2 / 3$. On the other hand, this is not true for somewhat higher densities: If $p \geq \lambda(\log n)^{3} /(n \log \log n)$ with $\lambda>1 / 8$ then with high probability we need to delete more edges than one from every triangle. Our result has a natural algorithmic interpretation.
\end{abstract}

*Keywords: random graph, cover graph, poset

Proposed running head: Sparse Cover Graphs

1991 Mathematics Subject Classification: 05C80, 06A07

${ }^{\dagger}$ Supported in part by NSF Grant DMS-9627408.

${ }^{\ddagger}$ Supported in part by NSF grant CCR-9530974.

$\S$ Permanent Address Computer and Automation Research Institute of the Hungarian Academy of Sciences, Budapest, P.O.Box 63, Hungary-1518. Supported in part by OTKA Grants T 030059 and T 29074 FKFP 0607/1999.

『Supported in part by NSF grant DMS-9970622. 
THE EleCtronic Journal of COMBinatorics 7 (2000), \#R19

\section{Cover Graphs}

The (Hasse) diagram of the finite poset $\mathcal{P}=(V, \prec)$ is the directed graph $\vec{G}=(V, A)$, where $(u, v) \in A$ iff $u \prec v$ and there is no $z \in V$ such that $u \prec z \prec v$. The finite undirected graph $G=(V, E)$ is a cover graph iff there exists an orientation of its edges $\vec{E}$ such that $\vec{G}=(V, \vec{E})$ is a diagram of some poset $\mathcal{P}=(V, \prec)$. Clearly, $\vec{G}=(V, A)$ is the diagram of a poset iff it contains no directed cycles and no directed quasicycles. A directed quasicycle is a cycle with oriented edges in which the reversal of the orientation of a single edge creates a directed cycle.

The relationship between cover graphs and graph parameters has been investigated in several papers. B. Descartes [5] (as noted in [2]) showed that there are cover graphs with arbitrarily large chromatic number and this was strenghtened by B. Bollobás [2] who showed that for every integer $k$ there is a lattice whose diagram has chromatic number at least $k$. Furthermore, it was proved by Nešetřil and Rödl [9] that there exist graphs which are not cover graphs and have arbitrarily large girth.

The triangle is not a cover graph, since every orientation of its edges results in either a directed cycle or quasicycle. However, after deleting an edge from it, we get a path of length two, which is already a cover graph. Obviously, if we delete sufficiently many edges from an arbitrary graph $G$ it will become a cover graph. Therefore, it is reasonable to ask, what is the minimum number $c(G)$ such that after deleting $c(G)$ edges from $G$ it will be a cover graph. This parameter was introduced by Bollobás, Brightwell, and Nešetřil [4].

First consider dense random graphs. It is shown in [4] that for arbitrary integer $l \geq 2$ and $p=p(n)=o\left(n^{(l-2) /(l-1)}\right), c\left(G_{n, p}\right) \leq(1+\delta) p n^{2} / 2 l \mathbf{w h p}^{1}$. Moreover, if $p=p(n)=n^{-1+\eta(n)}$, where $0<\eta_{0} \leq \eta(n) \leq 1$, then whp one needs to delete a positive proportion of edges from $G_{n, p}$ in order to get a cover graph. The auhors of [4] also conjectured that if $p n^{(l-2) /(l-1)} \rightarrow 0$ and $p n^{(l-1) / l} \rightarrow \infty$, then their upper bound gives the right constant, i.e., whp $c\left(G_{n, p}\right) \sim p n^{2} / 2 l$. This has been recently proved by Rödl and Thoma [10].

For sparse random graphs, the authors of [4] show that whp $c\left(G_{n, p}\right)=o\left(e\left(G_{n, p}\right)\right)$. Namely they prove the following two bounds. For every $c \geq 1$ there is a $b, b>$ $c \log (1+b)$, such that if $p=p(n) \leq \frac{c \log n}{n}$, then $c\left(G_{n, p}\right) \leq \frac{p n^{2}}{2} \cdot \frac{b}{c \log n}$. Further, for

\footnotetext{
${ }^{1} \mathrm{~A}$ sequence of events $\mathcal{E}_{n}$ occurs with high probability, whp, if $\operatorname{Pr}\left(\mathcal{E}_{n}\right)=1-o(1)$ as $n \rightarrow \infty$
} 
every $\delta>0$ and for every function $\omega=\omega(n)$, with $\omega \rightarrow \infty$ and $\omega=o\left(n^{\nu}\right)$ for every $\nu>0$, if $p=p(n) \leq \frac{\omega \log n}{n}$, then $c\left(G_{n, p}\right) \leq(1+\delta) \cdot \frac{p n^{2}}{2} \cdot \frac{\log \omega}{\log n}$.

For a graph $G$ let $\tau(G)$ denote the minimum number of edges that must be deleted in order to get a triangle-free graph. In this note we focus on the graph property $c(G)=\tau(G)$. Since a cover graph may contain no triangles $\tau(G) \leq c(G)$ always holds. We will show in this note, that for any constant $\kappa<2 / 3$ and $p \leq \kappa \log n / n$, $c\left(G_{n, p}\right)=\tau\left(G_{n, p}\right)$ whp (Theorem 1.1) while for any constant $\lambda>1 / 8$ and $p \geq$ $\lambda(\log n)^{3} /(n \log \log n), c\left(G_{n, p}\right)>\tau\left(G_{n, p}\right)$ whp (Theorem 1.2).

We may interpret our results in an algorithmic way. Consider a simple algorithm which takes a graph $G$ as input and deletes edges in copies of triangles as long as $G$ is not triangle-free. Then Theorem 1.1 implies that if the algorithm takes as input $G_{n, p}, p \leq \kappa \log n / n$ for a constant $\kappa<2 / 3$, then it outputs a cover graph whp. Note that the output graph will have whp girth equal to 4 . On a related note we point to [8] which surveys constructions of non cover graphs with a given girth. It is an open problem to construct small examples of non cover graphs with girth greater then 4 .

We will prove the following theorems:

Theorem 1.1 If $\kappa<2 / 3$ is constant and $p \leq \kappa \log n / n$ then $c(G)=\tau(G)$ whp.

Theorem 1.2 If $\lambda>1 / 8$ is constant and $p \geq \lambda(\log n)^{3} /(n \log \log n)$ then $c\left(G_{n, p}\right)>$ $\tau\left(G_{n, p}\right)$ whp.

For $p=c / n, c$ constant we know that the distribution of the number of triangles in $G_{n, p}$ is asymptotically Poisson with mean $c^{3} / 6,[6]$. So as an immediate corollary we get that if $p=c_{n} / n$ then

\section{Corollary 1.3}

$$
\lim _{n \rightarrow \infty} \operatorname{Pr}\left(G_{n, p} \text { is a cover graph }\right)= \begin{cases}1 & c_{n} \rightarrow-\infty \\ e^{-c^{3} / 6} & c_{n} \rightarrow c \\ 0 & c_{n} \rightarrow \infty\end{cases}
$$

Theorem 1.2 follows from the following stronger theorem:

Theorem 1.4 Suppose $\lambda>1 / 8$ is constant and $p \geq \lambda(\log n)^{3} /(n \log \log n)$ and $g \leq$ $\frac{\log n}{4 \log \log n}$. Let $H$ be obtained from $G_{n, p}$ by deleting all vertices which lie on a cycle of length at most $g$. Then whp $H$ is not a cover graph. 
THE ELECTROniC Journal of COMBinatorics 7 (2000), \#R19

Remark 1.5 Part of the results of [10] is based on a detailed analysis of the expansion properties of the random graph $G_{n, p}$. Using the same analysis of the expansion properties provides the same bound on $p$ in Theorem 1.4.

\section{Triangle-free graphs: proof of Theorem 1.1}

We will need the following lemma. Let $\chi=\chi(G)$ be the chromatic number of $G$. We will call a cycle of $G$ short if it contains $\leq \chi(G)$ vertices and long otherwise. As usual the distance between two sets $V_{1}$ and $V_{2}$ of vertices in $G$ is the length of the shortest path between $u \in V_{1}$ and $v \in V_{2}$.

Lemma 2.1 Let $G$ having the following properties:

(a) The distance between any two short cycles is at least $\chi+1$.

(b) No short cycle shares an edge with a cycle of length $\leq 2 \chi$.

Then $c(G)=\tau(G)$.

Proof Let $G^{\prime}=\left(V, E^{\prime}\right)$ be a triangle-free subgraph of $G$ which we get after deleting one edge of each triangle of $G$. Let $V_{1}, \ldots, V_{\chi}$ be a proper coloring of $G^{\prime}$ with $\chi$ colours. Define the orientation $\vec{G}^{\prime}=\left(V, \overrightarrow{E^{\prime}}\right)$ as follows. If $u \in V_{i}, v \in V_{j}$ and $i<j$ then orient the edge $\{u, v\}$ from $u$ to $v$. Notice that $\vec{G}^{\prime}$ is acyclic, i.e., there are no oriented cycles in $\overrightarrow{G^{\prime}}$. Also there are no long oriented quasicycles. Indeed, take an arbitrary long cycle $\mathrm{C}$ in $G^{\prime}$. Let $k=\max \left\{i: C \cap V_{i} \neq \emptyset\right\}$ and let $v \in C \cap V_{k}$. Both edges of $C$ incident to $v$ are oriented in $\overrightarrow{G^{\prime}}$ towards $v$. So in order to get an oriented cycle we must change the orientation of at least one of the $\operatorname{arcs}\left(u_{1}, v\right),\left(u_{2}, v\right)$ of $C$ which are incident with $v$. Assume we get an oriented cycle by reversing the arc $\left(u_{1}, v\right)$, say. Then the remaining edges of the cycle form an oriented path from $u_{1}$ to $v$ in $\vec{G}^{\prime}$ of length at least $\chi$, a contradiction.

Of course, we still may have short oriented quasicycles. We will change the above orientation as follows. If $\overrightarrow{G^{\prime}}$ contains no short quasicycles, then stop. If $\overrightarrow{G^{\prime}}$ contains a short quasicycle $\vec{C}$ then reverse an arc $(u, v)$ in $\vec{C}$ such that the resulting $\vec{C}^{\prime}$ is neither a directed cycle, nor a directed quasicycle. (Since $G^{\prime}$ is triangle-free, we can always do this.) Since G satisfies (a), i.e., in particular it contains vertex disjoint short cycles 
only, this process will terminate in at most $n / \chi$ steps and in the end there will be no short quasicycles.

We show now that we have created neither long cycles nor long quasicycles. Indeed, by (b), during the edge reversing process, we did not touch any long cycle having $\leq 2 \chi$ vertices. So take a long cycle $C$ having $\geq 2 \chi+1$ vertices. Let an edge $e \in C$ be short if it also lies on a short cycle and long otherwise. Long edges are unaffected by the reversing process of the previous paragraph. Label each short edge with the short cycle that produces it. Short edges with the same label form subpaths of $C$. By (a) the distance between any two short edges of $C$ with distinct labels is at least $\chi$ and there will be two paths in $C$ of length $\geq \chi$, made up long edges only. But the longest oriented path in $\vec{G}^{\prime}$ is of length $\chi-1$. So if there are two short edges with distinct labels then there are at least two long edges in $\vec{C}$ in both directions along the cycle. If the short edges of $C$ all have the same label and they make up more than one subpath then the paths of long edges in $C$ between these subpaths are of length at least $\chi$, else (b) is violated. Hence, again, there are at least two long edges in $\vec{C}$ in both directions along the cycle. Finally, suppose that the short edges of $C$ all have the same label and they make up one subpath $P$. If none of the edges of $P$ are reversed then by its length $C$ will have at least three edges oriented in each direction around $C$. In any case, at most one will be reversed and so we will create neither a directed cycle nor a directed quasicycle by the reversing process.

We complete the proof of Theorem 1.1 with

Lemma 2.2 If $\kappa<2 / 3$ is constant and $p \leq \kappa \log n / n$ then $G_{n, p}$ whp satisfies the conditions of Lemma 2.1.

Proof It follows from Euczak [7] that whp

$$
\chi\left(G_{n, p}\right) \leq k_{0}=\left(\frac{\kappa}{2}+o(1)\right) \frac{\log n}{\log \log n} .
$$

Assuming this we see that if the conditions of Lemma 2.1 are violated then there exists a set of $k \leq 3 k_{0}$ vertices which contain at least $k+1$ edges. The probability of 
this is at most

$$
\begin{aligned}
& \sum_{k=4}^{3 k_{0}}\left(\begin{array}{l}
n \\
k
\end{array}\right)\left(\begin{array}{c}
k \\
2 \\
k+1
\end{array}\right)\left(\frac{\kappa \log n}{n}\right)^{k+1} \leq \sum_{k=4}^{3 k_{0}}\left(\frac{n e}{k}\right)^{k}\left(\frac{k e}{2} \cdot \frac{\kappa \log n}{n}\right)^{k+1} \\
& \leq \frac{e \kappa \log n}{2 n} \sum_{k=4}^{3 k_{0}} k\left(\left(e^{2} \kappa / 2\right) \log n\right)^{k} \\
& \leq \frac{3 k_{0}\left(\left(e^{2} \kappa / 2\right) \log n\right)^{3 k_{0}+1}}{n} \\
& =o(1) \text {. }
\end{aligned}
$$

\subsection{Sparse Non Cover Graphs: proof of Theorem 1.4}

For positive integers $a, b$, a partition $V_{0}, V_{1}, \ldots, V_{a}$ of an $n$-set is called an $(a, b)$ partition if $\left|V_{0}\right|=b$ and $\left|V_{i}\right|=\left|V_{j}\right|$ for all $i \neq j ; i, j \in[a]$. Thus we can only have an $(a, b)$-partition if $a$ divides $|V|-b$.

We choose functions $d=d(n), \omega=\omega(n)$, and $N=N(n)$ such that

$$
\begin{aligned}
N & =n-\gamma=n-o(n) \\
\left(\frac{d}{\omega}\right)^{\omega} & =2 N \log \omega \\
d & =\omega^{3}(\log \omega)^{2}
\end{aligned}
$$

where $\gamma$ is the smallest integer larger than $g d^{g}$ such that $n-\gamma$ is divisible by $\omega$. Note that this choice implies $\omega=(1+o(1)) \frac{\log n}{2 \log \log n}$ and $d=(1+o(1)) \frac{(\log n)^{3}}{8 \log \log n}$.

Let $p=d / n$. Since the property in Theorem 1.4 is monotone decreasing it is enough to prove Theorem 1.4 just for this choice of $p$.

We need the following lemma.

Lemma 2.3 Whp for every $(\omega, \gamma)$-partition $V_{0}, V_{1}, \ldots, V_{\omega}$ of the vertex set of $G_{n, p}$, there is a cycle $C$ in $G_{n, p}$ such that $V(C) \cap V_{0}=\emptyset$ and $\left|V(C) \cap V_{i}\right|=1, i=1, \ldots, \omega$.

Proof To prove the lemma we are going to use the Janson inequality, cf. [1] for notation. Note that the number of choices of $(\omega, \gamma)$-partitions of an $n$-set is at most $\left(\begin{array}{c}n \\ \gamma\end{array}\right)(e \omega)^{N}$. 
Let $V_{0}, V_{1}, \ldots, V_{\omega}$ be a fixed $(\omega, \gamma)$-partition. Let $C_{1}, \ldots, C_{l}$ be an enumeration of cycles satisfying $V\left(C_{i}\right) \cap V_{0}=\emptyset$ and $\left|V\left(C_{i}\right) \cap V_{j}\right|=1, i=1, \ldots, l, j=1, \ldots, \omega$, in the complete graph $K_{n}$ with the partition $V_{0}, V_{1}, \ldots, V_{\omega}$. Set $\mathcal{B}_{i}, i=1, \ldots, l$, to be the event that $C_{i}$ exists in $G_{n, p}$. The expected number $\mu$ of such cycles in $G_{n, p}$ satisfies

$\mu=\sum_{j=1}^{l} \operatorname{Pr}\left(\mathcal{B}_{j}\right)=(1+o(1))\left(\frac{N}{\omega}\right)^{\omega}\left(\frac{d}{N}\right)^{\omega}=(1+o(1))\left(\frac{d}{\omega}\right)^{\omega}=(1+o(1)) 2 N \log \omega$

and

$$
\begin{aligned}
\Delta & =\sum_{\left|E\left(C_{i}\right) \cap E\left(C_{j}\right)\right| \geq 1} \operatorname{Pr}\left(\mathcal{B}_{i} \wedge \mathcal{B}_{j}\right) \\
& \leq \sum_{\substack{t \geq 1 \\
2 \leq L<\omega}} \sum_{\substack{l_{1}+\ldots+l_{t}=L \\
l_{i} \geq 2}}(1+o(1)) \omega^{t}\left(\frac{N}{\omega}\right)^{L}\left(\frac{N}{\omega}\right)^{2 \omega-2 L}\left(\frac{d}{N}\right)^{2 \omega-(L-t)} \\
& =(1+o(1)) \mu \cdot \sum_{t, L} \sum_{l_{1}, \ldots, l_{t}}\left(\frac{d}{\omega}\right)^{\omega} \frac{\omega^{L+t}}{d^{L-t} N^{t}} \\
& \leq(1+o(1)) \mu \cdot \sum_{t, L}\left(\begin{array}{l}
L-1 \\
t-1
\end{array}\right) 2 N \log \omega \cdot\left(\frac{\omega}{d}\right)^{L}\left(\frac{\omega d}{N}\right)^{t} \\
& =(1+o(1)) \mu \cdot \sum_{2 \leq L<\omega} 2 N \log \omega \cdot\left(\frac{\omega}{d}\right)^{L} \frac{\omega d}{N}\left(1+\frac{\omega d}{N}\right)^{L-1} \\
& =o(\mu) .
\end{aligned}
$$

Note that the dominant term in the last sum is the term for $L=2$.

Explanation of (1): The common edges of cycles $C_{i}, C_{j}$ are asumed to form $t$ paths of lengths $l_{1}, l_{2}, \ldots, l_{t}$. $\omega^{t}$ estimates the number of choices for the start vertices of these paths. $(N / \omega)^{L}$ estimates the choices for the vertices of these paths and $(N / \omega)^{2 \omega-2 L}$ estimates the choices for the vertices in $C_{i}, C_{j}$ which are not on any common path. $(d / N)^{2 \omega-(L-t)}$ is the probability that $C_{i}, C_{j}$ exist.

Applying the Janson inequality we see that

$\operatorname{Pr}(\exists$ an $(\omega, \gamma)$-partition without a cycle $) \leq\left(\begin{array}{c}n \\ \gamma\end{array}\right)(e \omega)^{N} e^{-(1-o(1)) \mu} \leq\left(\begin{array}{c}n \\ n^{3 / 4}\end{array}\right) \omega^{-(1-o(1)) N}=o(1)$.

We can assume $g=\left\lfloor\frac{\log n}{4 \log \log n}\right\rfloor$ and that the condition in Lemma 2.3 holds. Now 
the expected number of vertices $\nu$ on cycles of length $g$ or less in $G_{n, p}$ is given by

$$
\sum_{k=3}^{g}\left(\begin{array}{l}
n \\
k
\end{array}\right)(k-1) ! p^{k} \leq d^{g} \sum_{k=3}^{g} \frac{1}{k} \leq d^{g} \log g .
$$

So whp $\nu \leq g d^{g}$.

Let $\hat{V}_{0}$ be the set of vertices of $G$ incident to cycles of length $g$ or less and $V_{0} \supseteq \hat{V}_{0}$ be arbitrarily chosen of size $\gamma$. Then $G^{\prime}:=G \backslash V_{0}$ is not a cover graph. Suppose it is. Let $\vec{G}^{\prime}$ be its orientation as a diagram of some poset. Thus, we can embed $\vec{G}^{\prime}$ into a linear ordering $\pi$. Let $V_{1}, \ldots, V_{\omega},\left|V_{i}\right|=N / \omega$, be the partition of the vertex set of $G^{\prime}$ such that all vertices of $V_{i}$ precede all vertices of $V_{i+1}$ in the linear ordering $\pi, i=1, \ldots, \omega-1$. We have constructed an $(\omega, \gamma)$-partition $V_{0}, V_{1}, \ldots, V_{\omega}$ of the vertex set of $G$. By the lemma above we can assume that there is a cycle $C$ such that $V(C) \cap V_{0}=\emptyset$ and $\left|V(C) \cap V_{i}\right|=1, i=1, \ldots, \omega$. The cycle $C$ induces a quasicycle in $\overrightarrow{G^{\prime}}$ - contradiction. This completes the proof of Theorem 1.4.

\section{References}

[1] N. Alon, J. Spencer, The Probabilistic Method, John Wiley \& Sons Inc., New York, 1992.

[2] B. Bollobás, Colouring lattices, Alg. Universalis 7 (1977), 313-314.

[3] B. Bollobás, Random Graphs, Academic Press 1985.

[4] B. Bollobás, G. Brightwell and J. Nešetřil, Random Graphs and Covering Graphs of Posets, Order 3 (1986) 245-255.

[5] B. Descartes, A Three Color Problem, Eureka, April 1947. Solution March 1948.

[6] P.Erdős and A.Rényi, On the evolution of random graphs, Publ. Math. Inst. Hungar. Acad. Sci. 5 (1960) 17-61.

[7] T.Łuczak, The chromatic number of random graphs, Combinatorica 11 (1991) $45-54$. 
THE ELECTRONiC JOURNAL OF COMBINATORICS 7 (2000), \#R19

[8] O. Pretzel, Orientations and Edge Functions on Graphs, in: A. D. Keedwell (ed.), Surveys in Combinatorics, London Math. Soc. Lecture Notes 66, 1991, $161-185$.

[9] J. Nešetřil and V. Rödl, On a Probabilistic Graph-Theoretical Method, Proceedings AMS 72(2) (1978), 417-421.

[10] V. Rödl, L. Thoma, On Cover Graphs and Dependent Arcs in Acyclic Orientations, submitted. 